\title{
Association between oral candidiasis and low CD4+ count among HIV positive patients in Hoima Regional Referral Hospital
}

\author{
Martina Nanteza ${ }^{1 \dagger}$, Jayne B Tusiime ${ }^{2 \dagger}$, Joan Kalyango ${ }^{1,3+}$ and Arabat Kasangaki $i^{*^{*}+}$
}

\begin{abstract}
Background: The aim of this study was to determine the prevalence of Human Immune Virus (HIV) related oral lesions and their association with Cluster of Differentiation $4\left(\mathrm{CD}^{+}\right)$count among treatment naïve HIV positive patients.

Methods: This was a descriptive and analytical cross sectional study. Participants were 346 treatment naïve HIV positive adult patients. These were consecutively recruited from Hoima Regional Referral hospital between March and April 2012. Data collection involved interviews, oral examinations and laboratory analysis.

Results: A total of 168(48.6\%) participants had oral lesions. The four commonest lesions were oral candidiasis (24.9\%, Cl=20.6-29.7\%), melanotic hyperpigmentation (17.3\%, Cl=13.7-21.7\%), kaposi sarcoma (9.3\%, Cl=6.6-12.8\%) and Oral Hairy Leukoplakia $(\mathrm{OHL})(5.5 \%, \mathrm{Cl}=3.5-8.4 \%)$. There was significant association between oral candidiasis and immunosuppression measured as CD4+ less than 350 cells $/ \mathrm{mm}^{3}(\mathrm{OR}=2.69, \mathrm{Cl}=1.608-4.502, \mathrm{p}<0.001)$. Oral candidiasis was the only oral lesion significantly predictive of immunosuppression ( $\mathrm{OR}=2.56, \mathrm{Cl}=1.52-4.30, \mathrm{p}<0.001)$ with a Positive Predictive Value (PPV) of $48.2 \%$, Negative Predictive Value (NPV) of $74.3 \%, 38.1 \%$ sensitivity and specificity of $81.4 \%$.
\end{abstract}

Conclusion: Oral candidiasis can be considered as a marker for immunesuppression, making routine oral examinations essential in the management of HIV positive patients.

Keywords: Oral candidiasis, Low CD4+ count, HIV positive patients

\section{Background}

HIV is a major global health problem. Sub-Saharan Africa continues to bear an inordinate share of the global HIV burden with 23 million people living with HIV/AIDS residing in this region [1]. Current HIV prevalence rates in Uganda stand at 7.4\% [2] Among the HIV-associated infections, oral lesions have been recognized as prominent features since the beginning of the epidemic and continue to be important. Approximately 40-50\% of people who are HIV-positive have been reported to have oral fungal, bacterial or viral infections, which often occur early in the course of HIV infection [3]. The occurrence of these oral lesions in HIV infection reflects the immune status of the patient with many being associated with reduced $\mathrm{CD} 4+\mathrm{T}$ lymphocyte cell count. Because of this, they can be used as entry or end-

\footnotetext{
*Correspondence: arabat55@yahoo.com

${ }^{\dagger}$ Equal contributors

${ }^{4}$ Department of Dentistry, Makerere University, P.O. Box 33019, Kampala, Uganda Full list of author information is available at the end of the article
}

points in therapy and vaccine trials as well as in staging and classification systems $[4,5]$. They are thus not only important for their morbidity and mortality but also for their diagnostic value in monitoring the immune status of the patient.

In resource constrained settings, where routine access to immunological monitoring is limited, oral lesions have been suggested as useful aids that are complimentary to regular CD4+ count assessment [6]. In such settings therefore, monitoring is typically clinical and immunological, mainly as a result of financial and infrastructure constraints $[7,8]$. There are cardinal lesions that are strongly associated with HIV and used internationally in the disease staging. The prevalence of such lesions amongst HIV positive individuals varies from region to region. Agwu et al. [9] found a prevalence of $71 \%$ in south western Uganda, while Tirwome et al. [10] recorded a $72 \%$ prevalence among HIV patients in several TASO clinics. Given that disease patterns change over time, and differ according to region studied its vital to know the current 
prevalence rates in Hoima. Information regarding association between the oral lesions and immunesuppression among HIV positive patients in Uganda is lacking. The aim of this study therefore was to highlight the prevalence of oral lesions among HIV positive patients, in the long run expressing the oral disease burden among HIV patients. The relationship between these lesions and the immune status of the patients was assessed in order to show how, if coupled with immunological monitoring, patients in resource limited settings can have the disease properly staged, classified and managed.

\section{Methods}

\section{Participants and study setting}

This was a descriptive and analytical cross-sectional study carried out from March to April 2012, in which quantitative data collection methods were used. It was carried out among consenting treatment naïve HIV positive male and female patients aged 18 years and above attending Hoima Regional Referral hospital HIV clinic. Patients who were too ill to participate in the interviews and oral examinations were excluded. Participants $(\mathrm{n}=$ 346) were consecutively selected as they presented to the clinic. Using an interviewer administered, standard and pre-tested questionnaire, information on sociodemographic characteristics; socio-behavioral factors; experience with oral lesions, and their consequences was collected. Information about history of systemic comorbidities, history of previous HIV oral lesions, and prescribed medication was obtained from the patient's medical records.

\section{Investigations}

Oral examinations were carried out by a dental surgeon, who was blinded to the clinical staging and CD4+ count results of the patients. Patients were examined while seated in a chair and in a well illuminated room. The extra oral and perioral areas were examined first, followed by intraoral tissues for any abnormalities. For better exploration of the mouth, periodontal probes, dental explorers and dental mirrors were used. Diagnosis of the oral lesions was made using European Community (EC) clearing house guidelines for presumptive diagnosis of oral lesions [11]. According to these guidelines, the lesions are classified into 3 groups: those strongly associated with HIV infection; those less commonly associated with HIV infection; and those seen in, but not indicative of HIV infection.

Lesions diagnosed were recorded into the World Health Organization (WHO) recording form for oral lesions associated with HIV infection. The inner and buccal surfaces of the lower incisors and the first two molars were assessed for presence of plaque or calculus. These were the lower incisors and the first two upper molars, whose inner and buccal surfaces were respectively examined. A photo atlas was developed to document the lesions diagnosed during the study. The clinical staging of new patients was done by the medical doctors in the HIV clinic.

For the regular patients, the clinical staging was retrieved from the patient's records. The blood samples were obtained on the same day as the oral examinations and their results were recorded onto each participant's questionnaire.

\section{Data management and quality control}

Pretested questionnaires were used in data collection and were checked daily for consistency and completeness before the data was entered into the computer. Translation of the questionnaire into Runyakitara was done to cater for participants not well conversant with English. Data was entered in Epi data version 3.1, edited and cleaned and then exported to STATA version 9 for analysis.

\section{Data analysis}

Descriptive analysis was used to summarize the participants' characteristics using medians, percentages, frequencies and interquartile ranges. Odds ratios at 95\% confidence intervals were used to measure association between each of the predictor variables and presence of oral lesions. Chi-square test with level of significance at 0.05 was used to test the significance of the association between presence of oral lesions and the predictor variables. CD4 ${ }^{+}$was categorized into $\mathrm{CD} 4^{+}<350$ cells $/ \mathrm{mm}^{3}$ and $\mathrm{CD}_{4}^{+} \geq 350$ cells $/ \mathrm{mm}^{3}$, with immune suppression being defined as $\mathrm{CD} 4^{+}<350$ cells $/ \mathrm{mm}^{3}$ [12]. Positive and negative predictive values of the most common lesions for $\mathrm{CD} 4^{+}<350$ cells $/ \mathrm{mm}^{3}$ were computed. The sensitivity and specificity of the commonest lesions to diagnose immune suppression of $<350$ cells $/ \mathrm{mm} 3$ were determined. Variables with p-value less than 0.2 at bivariate analysis were considered for inclusion in the multiple logistic regression models, with presence of oral lesions as the outcome. With $\mathrm{CD} 4+$ as the main predictor, other predictors were assessed for confounding and interaction.

\section{Ethical considerations}

Permission to carry out the study was sought from Makerere University School of Medicine Research and Ethics Committee, the Uganda National Council of Science and Technology and Hoima hospital authorities. Written informed consent was obtained from the participants, and confidentiality was assured by concealing the patients' names and using identification numbers on the questionnaires. Participants diagnosed with different oral lesions were treated in Hoima hospital and others who required further management were referred to the relevant health units. 


\section{Results}

Of the 346 consenting participants, 265(76.6\%) were females and $81(23.4 \%)$ males (Table 1$)$. The median age in this study group was 33 years (IQR, 25-41). Of the participants, $124(35.8 \%)$ had a history of regular alcohol consumption and $25(7.2 \%)$ had a history of regular tobacco smoking. About 24 (7\%) of the respondents said they did not regularly brush their teeth, while $79(22.8 \%)$ reported brushing more than once daily. More than half of the respondents $(53.2 \%, \mathrm{n}=184)$ reported never having gone for a dental checkup, and 154 (44.5\%) reported going only when in discomfort. Most respondents had CD4+ count greater than 350 cells $/ \mathrm{mm}^{3}(88.1 \%, \mathrm{n}=296)$, and majority of the respondents belonged to WHO stage two, $(73.0 \%, \mathrm{n}=252)$.

HIV associated oral lesions were observed in $48.6 \%$ (CI = 43.3-53.8\%) ( $n=168)$ respondents. Among the 346 respondents, the four most common lesions were oral candidiasis,

Table 1 Sociodemographic, sociobehavioral, dentobehavioral and clinical characteristics of 346 participants from Hoima Regional Referral Hospital

\begin{tabular}{lll}
\hline Characteristics & Number & Proportion (\%) \\
\hline Sex &
\end{tabular}

Male

Female

Smoking

Yes

No

Alcohol consumption

Yes

No

Brushing frequency

Not often

Once daily

More than once daily

Dental visits

Never

Once a year

Every six months

When in discomfort

CD4* $(n=336)$

$<350$

$\geq 350$

WHO stage

\begin{tabular}{lcc} 
I & 75 & 21.7 \\
III & 252 & 72.8 \\
IV & 19 & 5.5 \\
\hline
\end{tabular}

melanotic hyperpigmentation, Kaposi sarcoma and OHL, in that order, as shown in Table 2 below. Oral candidiasis was present in $86(24.9 \%)$ of the respondents, with the pseudomembranous subtype being the most prevalent and present among 42 respondents (12\%). Melanotic hyperpigmentation was observed among 60 patients $(17.3 \%, \mathrm{CI}=$ 13.7-21.7\%), whereas kaposi sarcoma was diagnosed among 32 participants $(9.3 \% \mathrm{CI}=6.6-12.8 \%)$. Oral hairy leukoplakia was the least prevalent at $5.5 \%(\mathrm{n}=19, \mathrm{CI}=3.5-8.4 \%)$.

At bivariate analysis, CD4+ count of less than 350 cells $/ \mathrm{mm} 3$, was found to be significantly associated with presence of oral candidiasis $(\mathrm{OR}=2.691, \mathrm{p}<0.001, \mathrm{CI}=$ 1.608-4.502). In the multivariate model, CD4+ count, problems with oral functions and WHO stage were significantly associated with the presence of oral candidiasis as shown in Table 3.

In the multivariate model, after analysis, we did not find any confounding by any of the dependent variables, and neither was there any interaction between them. Candidiasis was the only significant predictor of immune suppression measured as CD $4<350$ cells $/ \mathrm{mm}^{3},(\mathrm{OR}=$ 2.56, 1.52-4.30, $\mathrm{p}<0.001$ ), (Table 4) The PPV of OHL, kaposi sarcoma, melanotic hyperpigmentation and candidiasis were $47.4 \%, 41.9 \%, 32.2 \%$ and $48.2 \%$ respectively. The NPV of OHL, kaposi sarcoma, melanotic hyperpigmentation, and candidiasis were 69.7\%, 69.8\%, 68.9\%, and $74.3 \%$ respectively. The sensitivity values were $8.6 \%$, $12.4 \%, 18.1 \%$ and $38.1 \%$ for OHL, kaposi sarcoma, melanotic hyperpigmentation and candidiasis respectively. Values for specificity were $95.7 \%, 92.2 \%, 82.7 \%$ and $81.4 \%$

Table 2 Prevalence of oral lesions among 346 Hoima hospital respondents

\begin{tabular}{lccc}
\hline Oral lesion & Frequency & Percentage & $\mathbf{9 5 \% ~ C l}$ \\
\hline Any oral lesion & 168 & 48.6 & $43.3-53.8$ \\
Oral candidiasis & 86 & 24.9 & $20.6-29.7$ \\
$\quad$ Pseudo membranous & 42 & 12.1 & $9.1-16.0$ \\
$\quad$ candidiasis & & & \\
$\quad$ Erythematous candidiasis & 29 & 8.4 & $5.9-11.8$ \\
$\quad$ Angular cheilitis & 15 & 4.3 & $2.7-7.0$ \\
Oral hairy leukoplakia & 19 & 5.5 & $3.5-8.4$ \\
Kaposi sarcoma & 32 & 9.3 & $6.6-12.8$ \\
Melanotic hyperpigmentation & 60 & 17.3 & $13.7-21.7$ \\
Linear gingival erythema & 7 & 2.0 & $0.9-4.1$ \\
Herpes simplex & 6 & 1.7 & $0.8-3.7$ \\
Recurrent apthous ulcers & 5 & 1.5 & $0.6-3.3$ \\
Xerostomia & 5 & 1.5 & $0.6-3.3$ \\
Salivary gland enlargement & 13 & 3.8 & $2.2-6.3$ \\
Rampant caries & 15 & 4.3 & $2.7-7.0$ \\
Calculus/plaque deposits & 18 & 5.2 & $3.3-8.1$ \\
\hline
\end{tabular}


Table 3 Results of multivariate analysis of factors associated with the presence of oral candidiasis

\begin{tabular}{llll}
\hline Variable & OR & p-value & $\mathbf{9 5 \% ~ C l}$ \\
\hline CD4+ & 0.997 & $<0.001$ & $0.995-0.999$ \\
Problem with oral functions & & & \\
$\quad$ None & 1.000 & & \\
$\quad$ Chewing & 7.389 & $<0.001$ & $3.391-16.102$ \\
WHO stage & & & \\
$\quad$ Stage 1 & & & \\
$\quad$ Stage 3 & 1.000 & & $1.182-12.240$ \\
\hline
\end{tabular}

for OHL, kaposi sarcoma, melanotic hyperpigmentation and candidiasis respectively.

\section{Discussion}

Prevalence of oral lesions

Since the beginning of AIDS epidemic, developing countries have experienced difficulties in implementing appropriate, inexpensive, and efficient HIV laboratory diagnostic techniques to aid in the epidemiological assessment and control of HIV infection. Studies have been conducted elsewhere for association of oral manifestations with absolute CD4 lymphocyte counts [13-15]. However, on doing a comprehensive literature search, there were no Ugandan studies for association of oral manifestations with CD4+ count. Hence an attempt was made in this study towards the correlation of oral manifestations with CD4+ count.

About five in every ten HIV patients in this study had oral lesions. The prevalence of oral lesions found in this study population was in agreement with the documented range of $30-80 \%$ seen in developing countries [16-19] and similar to findings from another study in Uganda [17]. It is however relatively lower than previously reported results from other studies done in different parts of Uganda where prevalence rates as high as $73.2 \%$ and $72 \%$ were found $[9,10]$. This discrepancy is probably due to the fact that there has been increased support from government and donor agencies, and better strategies for management of HIV over the years.

Oral candidiasis was the commonest lesion observed in this study population, agreeing with findings by Bodhade et al. (39.3\%), Kardpon et al. (55\%), Adrogubangba et al. (33.3\%) and Allan et al. (17.7\%) [12,15,20,21]. The pseudomembranous subtype was the commonest, just as was observed in south western Uganda (Table 2). Melanotic hyperpigmentation, although classified as a lesion less commonly associated with HIV, was the second most prevalent in this study. As the study group comprised of treatment naive patients, the probability that this high prevalence was due to effects of antiretroviral therapy is very small. From their records no participants had previously been staged as stage four patients though surprisingly, Kaposi sarcoma which designates an individual to WHO stage four of HIV disease, was found in 32 (9.3\%) of the participants. This exposes the discrepancies that occur in staging, and ultimately in management of HIV patients. Such discrepancies are more pronounced in resource limited settings where access to routine immunological monitoring is limited. Oral hairy leukoplakia had a low prevalence in this study, $(5.5 \%, \mathrm{n}=19)$ probably due to the fact that definitive diagnosis requires demonstration of Epstein- Barr virus, which wasn't conducted in this study and might have been a source of potential misclassification.

\section{Oral candidiasis in the prognosis of the disease}

Findings showed that individuals under WHO stage three are almost 4 times as likely as individuals in stage one to develop oral candidiasis (Table 3). Oral candidiasis is currently being used to stage patients as stage three under the WHO clinical staging system, making these findings scientifically sound. In this study, HIV positive patients with difficulty in chewing are seven times more likely to have oral candidiasis than those without any difficulty in chewing. When a comprehensive dental history is taken, it is possible to elicit such symptoms, which when followed with an oral examination will aid in the identification and diagnosis of oral lesions. The oral lesions are in turn used to diagnose and monitor immunesuppression.

\section{Association between oral lesions and CD4+ count less than 350 cells $/ \mathrm{mm} 3$}

Oral candidiasis was the only lesion found to have a significant association with CD4+ count of less than 350 cells $/ \mathrm{mm} 3,(\mathrm{OR}=2.691, \mathrm{p}<0.001, \mathrm{CI}=1.608-4.502)$. Thus in this study, immunosuppressed individuals were almost thrice as likely to have oral candidiasis as their immune competent counterparts. In addition, oral candidiasis was the only lesion significantly predictive of immunesuppression in this study (Table 4). The

Table 4 Association of oral lesions with immune suppression (CD4 $<350$ cells $/ \mathrm{mm}^{3}$ )

\begin{tabular}{lccccccc}
\hline Oral lesion & OR & $\mathbf{9 5 \% ~ C l}$ & p-value & PPV & NPV & Sensitivity & Specificity \\
\hline OHL & 1.79 & $0.68-4.67$ & 0.238 & 47.4 & 69.7 & 8.6 & 95.7 \\
Kaposi sarcoma & 1.48 & $0.67-3.27$ & 0.334 & 41.9 & 69.8 & 12.4 & 92.2 \\
Melanotic hyper pigmentation & 0.96 & $0.51-1.81$ & 0.896 & 32.2 & 68.9 & 18.1 & 82.7 \\
Candidiasis & 2.56 & $1.52-4.30$ & $<0.001$ & 48.2 & 74.3 & 38.1 & 81.4 \\
\hline
\end{tabular}


findings imply that individuals with oral candidiasis are thrice as likely to be immunosuppressed as those without candidiasis. Therefore, oral candidiasis can be used as an index for immunosuppression depicted by CD4+ less than 350 cells in this study population.

These findings can be projected to other resource constrained settings, which makes routine oral examinations an essential part of management of HIV patients in these settings.

PPVs for oral manifestations to CD4+ less than 350 cells $/ \mathrm{mm} 3$ were in a low range from $32.2 \%$ for melanotic hyperpigmentation to $48.2 \%$ for candidiasis (Table 4 ). Thus, with oral candidiasis as a test, forty eight (48) out of a hundred (100) HIV patients diagnosed as beingimmunosuppressed will truly be immunosuppressed. This value is much lower than that from studies by Patton et al. (60.3\%) and Glick et al. (69.9\%) [22,23] owing to the low prevalence of immunosuppression in this study. The range for NPV was from $68.9 \%$ for melanotic hyperpigmentation to $74.3 \%$ for oral candidiasis Because the prevalence of immunosuppression in this population was low, the high NPV value of $74.3 \%$ for oral candidiasis makes it a not very informative test as regards to diagnosing the immunosuppression. Oral hairy leukoplakia had the highest specificity, of $95.7 \%$. This means that when used as a test for immunesuppression, the likelihood for the occurrence of false positive results is low. As is already the practice, oral hairy leukoplakia is being utilized in patient staging. What these results emphasize is the importance of oral examinations during routine patient management.

Oral candidiasis had the highest sensitivity of $38.1 \%$. Thus if oral candidiasis is used as a test, the proportion of individuals that would be correctly classified as immunosuppressed would be $68 \%$, making candidiasis a fairly good test for the diagnosis of immunosuppression in the study population. Generally, the presence of oral candidiasis should serve to herald the possibility of a compromised immunity amongst HIV patients, thus making oral examinations vital and beneficial towards the management of the patients.

\section{Limitations, conclusions and recommendations}

Diagnosis of the oral lesions was solely based on clinical findings, and no definitive diagnostic tests were performed. This might have introduced measurement bias into the study, in the form of non differential misclassification bias. However, the effect of this is likely to be minimal, as the diagnosis of the oral lesions followed an internationally accepted and recognized standard set of guidelines, the EC Clearing House guidelines. In addition, the significant odds ratios seen are actually true effect measures.
The study indicates that almost half in every one hundred study participants had an oral lesion, alone or in combination with other types, which is a moderately high rate.

The study demonstrated the possibility of oral candidiasis as an index to predict immunosuppression in resource limited settings with no access to regular CD4+ count monitoring. However to incorporate it as an index, certain regional parameters and research studies that employ rigorous research methodologies need to be done. There is a need to compare the results of various cross-sectional studies, and more longitudinal studies are required to formulate oral lesion indices that can help as markers for HIV-related immune suppression.

\section{Competing interests}

The authors declare that they have no competing interests.

\section{Authors' contributions}

Conceived and designed the experiment: MN JBT JK AK. Analyzed the data: MN JK. Wrote the paper: MN JBT AK. Revised the article: JBT JK AK.

Commented on the article: JBT JK AK. All authors read and approved the final manuscript.

\section{Acknowledgements}

This work was supported by Training Health Researchers into Vocational Excellence in East Africa (THRiVE), grant number 087540 funded by the Wellcome Trust. Its contents are solely the responsibility of the authors and do not necessarily represent the official views of the supporting offices. We are also most appreciative to the management of Hoima Regional Referral Hospital for granting us access to their facilities.

\section{Author details}

${ }^{1}$ Clinical Epidemiology Unit, Makerere University, Kampala, Uganda. ${ }^{2}$ School of Public Health, Makerere University, Kampala, Uganda. ${ }^{3}$ Department of Pharmacy, Makerere University, Kampala, Uganda. ${ }^{4}$ Department of Dentistry, Makerere University, P.O. Box 33019, Kampala, Uganda.

Received: 20 March 2014 Accepted: 13 November 2014 Published: 28 November 2014

\section{References}

1. USAID: HIV/AIDS health profile 2011; 2012.

2. Uganda AIDS Indicator Survey 2011: Uganda Aids Indicator Survey Preliminary Report; 2012.

3. World Health Organisation: Media Centre: Oral health; 2007.

4. Hodgson TA, Greenspan D, Greenspan JS: Oral lesions of HIV disease in industrialized countries. proceedings of the 5 th world workshop on oral health and disease in AIDS. Adv Dent Res 2005, 19:5-9.

5. Greenspan JS: Sentinels and signposts: the epidemiology and significance of the oral manifestations of HIV disease. Oral Dis 1997, 3:1.

6. Sharma G, Pai KM, Setty S, Ramapuram JT, Nagpal A: Oral manifestations as predictors of immune suppression in a HIV-/AIDS-infected population in south India. Clin Oral Investig 2009, 13:2.

7. World Health Organization (WHO): Antiretroviral therapy for HIV infection in adults and adolescents. In Recommendation for a public health approach; 2006.

8. Schooley Robert T: Viral load testing in resource-limited settings. Clin Infect Dis 2007, 44:1.

9. Agwu E, Ihongbe JC, Tirwomwe JF, Pazos V, Tirwomwe M, Casadesus L: Appraisal of oral lesions status of HIV/AIDS patients in South Western Uganda. Brazillian J Oral Sci 2008, 7:26.

10. Tirwomwe J, Rwenyonyi C, Muwazi L, Besigye B, Mboli F: Oral manifestations of HIV/AIDS in clients attending TASO clinics in Uganda. Clin Oral Investig 2007, 11:3.

11. Clearinghouse EC: WHO collaborating centre: oral problems related to hiv infection, oral manifestations of the human immunodeficiency virus. J Oral Pathol Med 1993, 22:289-291. 
12. Bodhade AS, Ganvir SM, Hazarey VK: Oral manifestations of HIV infection and their correlation with CD4 count. J Oral Sci 2011, 53:2.

13. Tsang PC, Samaranayake LP: Oral manifestations of HIV infection in a group of predominantly ethnic Chinese. J Oral Pathol Med 1999, 28:3.

14. Ranganathan K, Reddy BV, Kumarasamy N, Solomon S, Viswanathan R, Johnson NW: Oral lesions and conditions associated with human immunodeficiency virus infection in 300 south Indian patients. Oral Dis 2000, 6:3.

15. Kerdpon D, Pongsiriwet S, Pangsomboon K, lamaroon A, Kampoo K, Sretrirutchai S, Geater A, Robison V: Oral manifestations of HIV infection in relation to clinical and CD4 immunological status in northern and southern Thai patients. Oral Dis 2004, 10:3.

16. Arotiba JT, Adebola RA, lliyasu Z, Babashani M, Shokunbi WA, Ladipo MMA, Akhiwu BI, Osude OD: Oral manifestations of Hiv/Aids infection in Nigerian patients seen in Kano. Nigerian J Surg Res 2005, 1(7):176-181.

17. Mayanja B, Morgan D, Ross A, Whitworth J: The burden of mucocutaneous conditions and the association with HIV-1 infection in a rural community in Uganda. Trop Med Int Health 1999, 4:5.

18. Arendorf TM, Bredekamp B, Cloete CA, Sauer G: Oral manifestations of HIV infection in 600 South African patients. J Oral Pathol Med 1998, 27:176-179.

19. Patton LL, McKaig R, Strauss R, Rogers D, Eron JJJ: Changing prevalence of oral manifestations of human immuno-deficiency virus in the era of protease inhibitor therapy. Oral Surg Oral Med Oral Pathol Oral Radiol Endodontics 2000, 89:299-304.

20. Adurogbangba Ml, Aderinokun GA, Odaibo GN, Olaleye OD, Lawoyin TO: Oro facial lesions and CD4 counts associated with HIV/AIDS in an adult population in Oyo State, Nigeria. Oral Dis 2004, 10:319-326.

21. Lourenco AG, Moraes LT: Oral lesions in HIV infected individuals from Ribeirão Preto, Brazil. Med Oral Patholol Oral Cir Bucal 2008, 13:5.

22. Patton LL: Sensitivity, specificity, and positive predictive value of oral opportunistic infections in adults with HIV/AIDS as markers of immune suppression and viral burden. Oral Surg Oral Med Oral Pathol Oral Radiol Endod 2000, 90:182-188.

23. Glick M, Muzyka BC, Lurie D, Salkin LM: Oral manifestations associated with HIV - related disease as markers for immune suppression and AIDS. Oral Surg Oral Med Oral Pathol 1994, 77:4.

doi:10.1186/1472-6831-14-143

Cite this article as: Nanteza et al: Association between oral candidiasis and low CD4+ count among HIV positive patients in Hoima Regional Referral Hospital. BMC Oral Health 2014 14:143.

\section{Submit your next manuscript to BioMed Central and take full advantage of:}

- Convenient online submission

- Thorough peer review

- No space constraints or color figure charges

- Immediate publication on acceptance

- Inclusion in PubMed, CAS, Scopus and Google Scholar

- Research which is freely available for redistribution 